\title{
Improvement in the Neutrophil-Lymphocyte Ratio after Combined Fluorouracil, Leucovorina and Oxaliplatino based (FOLFOX) Chemotherapy for Stage III Colon Cancer is Associated with Improved Minimal Residual Disease and Outcome
}

\author{
Nigel P Murray ${ }^{1 *}$, Ricardo Villalon ${ }^{2}$, Dan Hartmann ${ }^{1}$, Patricia Maria Rodriguez ${ }^{2}$, \\ Socrates Aedo ${ }^{1}$
}

\begin{abstract}
Introduction: Minimal residual disease (MRD) is the net result of the biological properties of disseminated tumour cells and the effect of the immune system and treatment to eliminate them. The aim of this study was to analyse the effect of combined chemotherapy on the immune function as determined by the neutrophil-lymphocyte ratio (NLR) and if it was associated with changes in the subtype of minimal residual disease and outcome in stage III colon cancer. Methods and Patients: A prospective, single centre observational study; the NLR was determined immediately prior to and one, two and three months after completing chemotherapy. Circulating tumour cells (CTCs) and bone marrow micro-metastasis $(\mathrm{mM})$ using immunocytochemistry with anti-CEA were determined prior to and one month after chemotherapy. The association of changes in the NLR with MRD subtypes classified as Group I (negative for CTCs and $\mathrm{mM}$ ), Group II (positive for $\mathrm{mM}$ ) and Group III (positive for CTCs) as a result of chemotherapy and five-year disease free progression (DFS) analysed. Results: One hundred and eighty eight patients participated of whom 83 (44.9\%) relapsed. In non-relapsing patients the NLR significantly increased and was higher after chemotherapy compared with relapsing patients. Significant increases in the NLR were associated with changes to a better MRD prognostic subtype and decreases with a worse MRD subtype. Neither baseline NLR nor MRD subtype predicted response to chemotherapy. DFS for MRD subgroups were $88 \%, 56 \%$ and $6 \%$ for Groups I to III respectively. Conclusions: Immune function as measured by the NLR is associated with MRD prognostic subtypes, improvements in the NLR are associated with improvements in MRD post chemotherapy but neither baseline NLR or MRD predicted outcome.
\end{abstract}

Keywords: Colon cancer- minimal residual disease- immune dysfunction- neutrophil-lymphocyte ratio-chemotherapy

Asian Pac J Cancer Prev, 23 (2), 591-599

\section{Introduction}

The use of adjuvant chemotherapy after curative resection for stage III colon cancer is associated with a $33 \%$ decrease in the relative risk of relapse and an absolute survival benefit of 10-15\% (Salvatore et al., 2020). Current guidelines recommend that stage III patients should be treated with 5-fluorouracil (5-FU) and oxaliplatin (OX) based chemotherapy regimes within 4-8 weeks after curative resection (Shingawa et al., 2018). Chemotherapy is used to eliminate tumour cells that have disseminated prior to surgery; the presence of occult tumour cells is termed minimal residual disease (MRD). The presence of MRD depends on two principal factors; firstly the biological properties of the tumour cells within the primary tumour to disseminate, implant in distant tissues, survive and cause immunosuppression versus the effect of the immune response and treatment to eliminate them. Both innate and acquired host immune mechanisms may act during the differing stages of metastasis; eliminating tumour cells within the primary tumour, during their dissemination and in the metastatic niches. This interaction between tumour cells and the immune system is dynamic and may change with time. Clonal instability of cancer cells, selection of resistant cancer cells or the reduction of tumour load as a result of treatment may all modulate the immune response and as such the presence or absence of MRD.

The use of FOLFOX not only results in a direct cytotoxic effect on tumour cells (and normal cells) but 
also causes host immunomodulation (Gmeiner, 2020). After initial immunosuppression 5-FU causes a rebound response to restore steady state immune levels, increases tumour antigen visibility and thus effector T-cell responses and is cytotoxic to immunosuppressive myeloid derived suppressor cells (Li and Slayton, 2013; Gmeiner, 2020). Whereas oxaliplatin reduces immunosuppressive regulatory T-cells (Tregs) and increases activated cytotoxic T-cells (Stojanovsaka et al., 2019).

It has been proposed that the neutrophil-lymphocyte ratio is a measure of the equilibrium between the anti-tumour immune response and pro-tumour inflammation. (Maliietzis et al., 2014). The presence of an elevated NLR pre-resection has been associated with a worse disease free survival (DFS) and overall survival (OS) (Absenger et al., 2013; Shibutani et al., 2013; Maliietzis et al., 2014) and has been reported to be superior when compared to other inflammatory biomarkers (Song et al., 2017). There are few reports of the NLR determined post-resection, but these also report that an elevated NLR is associated with outcome and may be superior to pre-resection NLR values (Shibtani et al., 2015; Yasui et al., 2021). An elevated NLR has also been reported to predict a poor response to oxaliplatin-based chemotherapy (Kaneko et al., 2012).

We present a prospective observation study of the changes in the NLR en relation to the MRD sub-types and before and after FOLFOX chemotherapy for stage III colon cancer and patient outcome.

\section{Materials and Methods}

A single centre prospective observational study of consecutive patients with colon cancer referred for evaluation of minimal residual disease between January 2007 and December 2014. The study complied with the STROBE guidelines on cohort studies and included patients with pathological stage III colon cancer who had negative surgical margins, had no evidence of metastasis based on CT scanning of thorax, abdomen or pelvis and were to receive six cycles of FOLFOX chemotherapy. Exclusion criteria included; those patients who had received neo-adjuvant chemotherapy and those with multiple primary malignancies.

\section{Neutrophil to lymphocyte ratio}

A full blood count was taken immediately prior to the first cycle of chemotherapy and one month following completion of six cycles of FOLFOX. The absolute neutrophil and lymphocyte counts were determined (Siemens autoanalyzer) and the NLR calculated using the formula absolute neutrophil count/absolute lymphocyte count. Based on previously reported studies, NLR cut-off values of 3.0, 4.0 and 5.0 was used to analyse the differing parameters (Mallietzis et al., 2014; Borazan et al., 2017; Yasui et al., 2021).

\section{CTC detection}

At the same time as full blood count sampling, blood was taken to detect CTCs. The first $5 \mathrm{ml}$ was discarded to prevent possible epithelial cell contamination; the second $8 \mathrm{ml}$ was collected into EDTA containing tubes (Becton- Vacutainer $^{\circledR}$, USA). Specimens were processed within 24 hours and maintained at room temperature.

Differential gel centrifugation with Histopaque $1,077^{\circledR}$ (Sigma-Aldrich, USA) was used to obtain mononuclear cells according to the manufacturer's instructions and re-suspended in $100 \mu \mathrm{l}$ of autologous plasma. Four slides (sialinized, DAKO, USA) were prepared using $25 \mu$ l of cell suspension, dried and fixed in a solution of $70 \%$ ethanol, $5 \%$ formaldehyde and $25 \%$ phosphate buffered saline (PBS) pH 7.4 (DAKO, USA) for five minutes and finally washed twice with PBS.

Within one hour of fixation the specimens were incubated for one hour at room temperature with monoclonal anti-CEA clone 11-7 (DAKO, USA).

An alkaline phosphatase-anti-alkaline phosphatase based system (LSAB2, DAKO, USA) with neofuschin as the chromogen was used to detect CTCs. Positive samples underwent a second process using anti-CD45 clone 2B11 + PD7/26 (DAKO, USA); incubated for one hour at room temperature and identified using a peroxidase based system (LSAB2, DAKO, USA) with DAB (3,3'diaminobenzadine tetrachloride) as the chromogen.

A CTC was defined according to the morphological criteria of ISHAGE (Borgen et al., 1999), as a nucleated cell expressing CEA but not CD45. A positive test was defined as the detection of at least one cell $/ 8 \mathrm{ml}$ venous blood. (Figures 1 and 2)

\section{Bone marrow micro-metastasis detection}

A bone marrow biopsy was undertaken one month after surgery using midazolam as sedation and $2 \%$ lidocaine as anaesthesia local taken from the posterior superior iliac crest. Four "touch preps" using sialinized slides were made and processed as described for CTCs. Cells staining positive for CEA and negative for CD45 were defined as micro-metastasis (Figure 3 and 4).

Three prognostic sub-groups were formed; Group I negative for CTCs and bone marrow micro-metastasis (MRD negative), Group II positive for only bone marrow micro-metastasis (MRD positive), Group III CTC positive independent of bone marrow micro-metastasis status (MRD positive).

Study end point: The primary study end point was the association between the changes in the NLR and the three prognostic MRD groups one month after completing six cycles of FOLFOX. The secondary end point was the outcome of each group in terms of progression free survival.

\section{Statistical Analysis}

The analysis was performed using the program Stata (Stata/SE 14.0 for Windows, Stata Corp Lp, 20159). The mean and median were used to describe the clinical-pathological variables and the standard deviation and inter-quartile range (IQR) to measure dispersion. The frequencies of the differing variables were compared using the Chi-squared test. A NLR cut-off value of 3.0 and 5.0 was used to determine high and low risk patients and compare with the different clinical-pathological and MRD parameters. 
A $p$ value $<0.05$ was taken to signify statistical significance and all tests were two tailed. Kaplan-Meier analysis was used to determine disease free progression times for the whole cohort and MRD sub-groups. For five years of follow-up the restricted mean disease free progression survival time was determined for the whole cohort and MRD sub-groups.

\section{Results}

The observed cohort included 188 patients, 96 $(51.0 \%)$ males with a median follow-up time of 4.1 years (IQR: 3,8-4.4 years) with minimum and maximum, respectively, of 0.8 and 5.2 years. The median age was 68.7 years (IQR 53.8-83.6 years). All patients underwent 6 cycles of FOLFOX chemotherapy and none were lost to follow-up. A total of $82(43.0 \%)$ patients relapsed within the five years of follow-up.

For the whole cohort the median NLR pre-chemotherapy was 3.8 (IQR 2.5-6.0) versus 3.0 (IQR 2.1-4.9) one month post-chemotherapy $(\mathrm{p}<0.05) ; 2.7$ (IQR 2.0-5.2) two months post chemotherapy $(\mathrm{p}<0.03)$ and 2.7 (IQR 2.1$5.4)$ three months post chemotherapy $(p<0.05)$. Using NLR cut off values of 3.0, 4.0 and 5.0; the frequency of patients with a lower NLR was significantly higher post-chemotherapy for each cut off value (see Table 1).

The comparison of the NLR between patients who did not relapse versus those who relapsed

Comparing patients who relapsed versus those who did, the median NLR pre-chemotherapy were 4.6 (IQR 2.7-6.6) versus 3.5 (IQR 2.5-5.4) respectively ( $p=0.31$ ). The median NLR values one, two and three months postchemotherapy are shown in Table 2. The median NLR were significantly lower in patients who did not relapse versus those who relapsed for the three time periods, the median NLR was significantly lower post chemotherapy in patients who did not relapse compared with the prechemotherapy value $(p<0.001)$. In patients who relapsed there was no such significant difference $(p=0.55)$. The area under the curve to predict relapse using the NLR one-month post chemotherapy was 0.824 (considered to be good).

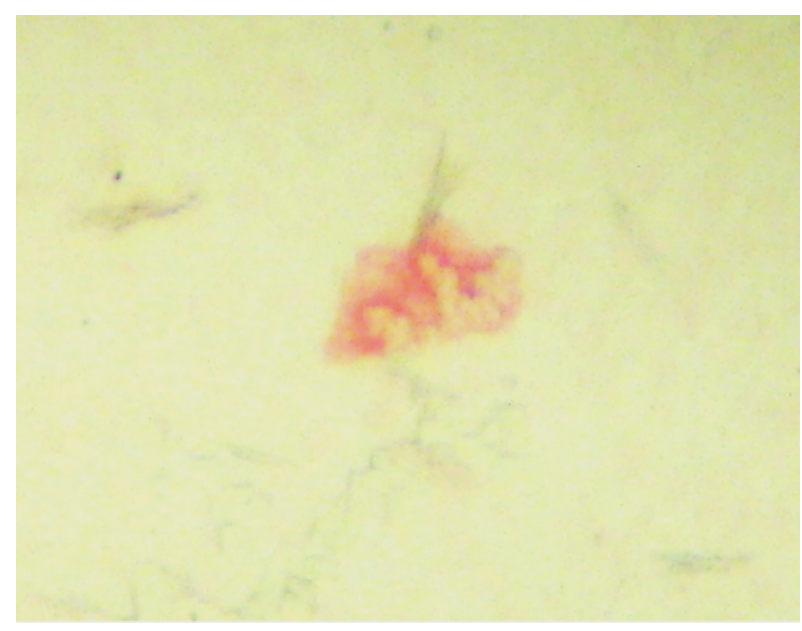

Figure 1. Two CTCs Staining Positive for CEA (red) and Negative for Membrane CD45.

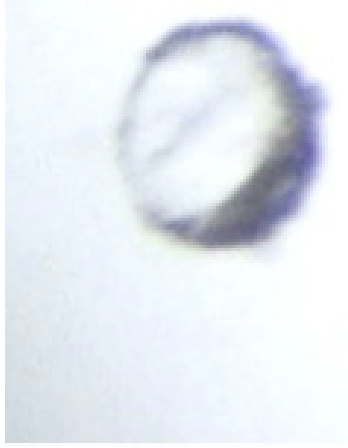

Figure 2. Leukocyte Negative for CEA and Ppositive for Membrane CD45 (Brown)

At all NLR cut off values the frequency of a lower NLR group was significantly higher in patients who did not relapse. In both patients who relapsed and did not relapse the frequency of a lower NLR for all cut off values was significantly higher post chemotherapy compared to the pre-chemotherapy values $(p=0.001$ and 0.0001 respectively). Although numerically the frequency of a lower NLR increased from one to three months in patients who did not relapse this did not reach statistical significance ( $\mathrm{p}=0.3$ ) except for the cut-off value of $<5.0$ $90 / 106$ versus $101 / 106(p=0.02)$. Similarly in patients who relapsed, only with a cut-off value of $<5$ was there a significant decrease 52/82 versus $36 / 46$ ( $p<0.02$ ).

\section{Subtypes of minimal residual disease and outcome}

Patients were divided into three groups, those patients negative for CTCs and micro-metastasis (Group I MRD negative), those only positive for micro-metastasis (Group II MRD positive) and those patients with CTCs detected irrespective of the presence of micro-metastasis (Group III MRD positive, CTC positive). Pre-chemotherapy 48 (25.9\%) patients formed Group I, 52 (28.1\%) Group II and $88(46.0 \%)$ Group III. The response to chemotherapy was heterogeneous and the subtype of MRD did not predict

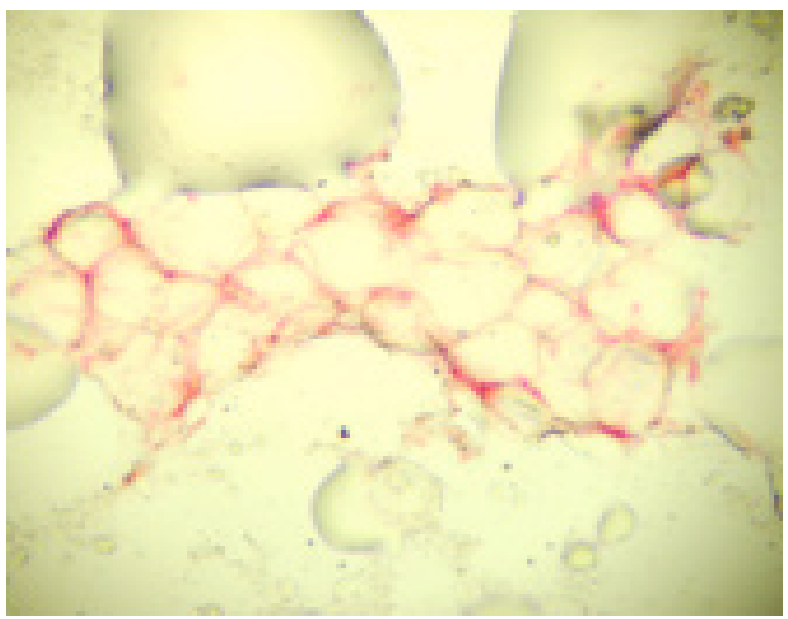

Figure 3. Bone Marrow Micro-Metastasis Staining Positive for CEA (Red) and Negative for Membrane CD45.

Asian Pacific Journal of Cancer Prevention, Vol 23 
Table 1. Neutrophil to Lymphocyte Ratio Pre and Post Chenotherapy for the Whole Cohort

\begin{tabular}{lcccc}
\hline & Pre-CT & 1 month post CT & 2 months post CT & 3 months post CT \\
\hline Whole cohort & $\mathrm{N}=188$ & $\mathrm{~N}=188$ & $\mathrm{~N}=188$ & $\mathrm{~N}=188$ \\
Median (IQR) & $3.8(2.5-6.0$ & $3.0(2.1-4.9)$ & $2.7(2.0-5.2)$ & $2.7(2.1-5.4)$ \\
& & $\mathrm{p}=<0.05$ & $\mathrm{p}>0.03$ & $\mathrm{p}<0.05$ \\
Neutrophil to lymohocyte ratio & & & & $102(54)$ \\
$<3.0 \mathrm{~N}(\%)$ & $68(36)$ & $94(50)$ & $98(52)$ & $86(46$ \\
$\geq 3.0 \mathrm{~N}(\%)$ & $120(64)$ & $94(50)$ & $90(48)$ & $\mathrm{p}=<0.001$ \\
& & $\mathrm{p}=0.009$ & $\mathrm{p}=0.003$ & $116(61)$ \\
$<4.0 \mathrm{~N}(\%)$ & $98(52)$ & $118(62)$ & $120(64)$ & $72(39)$ \\
$\geq 4.0 \mathrm{~N}(\%)$ & $90(48)$ & $70(38)$ & $68(36)$ & $\mathrm{p}>0.05$ \\
& & $\mathrm{p}<0.04$ & $138(73)$ & $136(72)$ \\
$<5.0 \mathrm{~N}(\%)$ & $114(60)$ & $142(75)$ & $50(27)$ & $52(28)$ \\
$\geq 5.0 \mathrm{~N}(\%)$ & $74(40)$ & $46(25)$ & $\mathrm{p}=0.01$ & $\mathrm{p}=0.02$ \\
\hline
\end{tabular}

CT, chemotherapy; IQR, inter-quartile range

the response (Table 1). Post-chemotherapy 95 (50.5\%) patients formed Group I, 37 (19.7\%) Group II and 56 (29.8\%) Group III.

The observed survival (Kaplan-Meier) at five years and the restricted mean survival times at five years are shown Figure 5 for the three MRD subtypes determined after chemotherapy. In terms of disease free progression, there was an increasing worse outcome and shorter times to progression from Group I to Group III (log-rank test $\mathrm{p}<0.01)$.

Association between the change in the median NLR with the change in MRD sub-type as a result of chemotherapy

Pre-chemotherapy the only significance difference in the median NLR between MRD subtypes was the lower median NLR in Group I (MRD negative) compared to Group III (CTC positive) $(\mathrm{p}<0.05)$. After chemotherapy

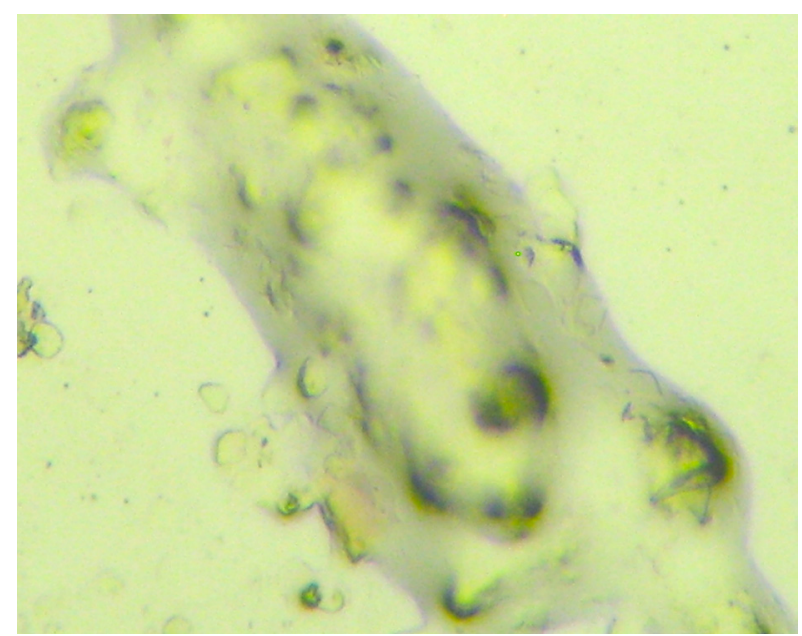

Figure 4. Bone Marrow Staining Negative for CEA

Table 2. Comparison of the Neutrophil to Lymphocyte Ratios and Frequency of Differing Cut-Offvalues in Patients Relapsedd and Did not Relapse

\begin{tabular}{|c|c|c|c|c|}
\hline Nuetrophil to lymphocyte traio & Pre CT & one month post $\mathrm{CT}$ & 2 months post $\mathrm{CT}$ & 3 months post $\mathrm{CT}$ \\
\hline \multicolumn{5}{|l|}{ Median (IQR) whole cohort) } \\
\hline Relapsed N = 82 & $4.6(2.7-6.6)$ & $4.6(3.4-5.5)$ & $4.9(3.8-7.5)$ & $5.4(3.9-7.9)$ \\
\hline \multirow[t]{2}{*}{ No relapse $\mathrm{N}=106$} & $3.5(2.5-5.4)$ & $2.3(1.9-2.9)$ & $2.2(1.8-2.6)$ & $2.2(1.8-2.7)$ \\
\hline & $\mathrm{p}=0.31$ & $\mathrm{p}<0.002$ & $\mathrm{p}<0.001$ & $\mathrm{p}<0.001$ \\
\hline \multicolumn{5}{|c|}{ Neutrophil to lymphocyte ratio $<3.0 \mathrm{~N}(\%)$} \\
\hline relasped $\mathrm{N}=82$ & $1(1)$ & $14(17)$ & $14(17)$ & $14(17)$ \\
\hline \multirow[t]{2}{*}{ No relapse $\mathrm{N}=106$} & $40(37)$ & $80(75)$ & $85(80)$ & $88(83)$ \\
\hline & $\mathrm{p}<0.0001$ & $\mathrm{p}<0.0001$ & $\mathrm{p}<0.0001$ & $\mathrm{p}<0.0001$ \\
\hline \multicolumn{5}{|c|}{ Nuetrophil to lymphocyte ratio < $4.0 \mathrm{~N}(\%)$} \\
\hline relasped $\mathrm{N}=82$ & $1(1)$ & $32(39)$ & $27(32)$ & $21(25)$ \\
\hline \multirow[t]{2}{*}{ Norelapse $\mathrm{N}=106$} & $62(58)$ & $88(83)$ & $94(88)$ & $95(89)$ \\
\hline & $\mathrm{p}<0.0001$ & $\mathrm{p}<0.0001$ & $\mathrm{p}<0.001$ & $\mathrm{p}<0.001$ \\
\hline \multicolumn{5}{|c|}{ Neutropil to lymphocyte ratio $<5 \mathrm{~N}(\%)$} \\
\hline relapsed $\mathrm{N}=82$ & $8(9)$ & $51(62)$ & $42(51)$ & $35(42)$ \\
\hline \multirow[t]{2}{*}{ No relapse N 0106} & $70(66)$ & $91(85 \%)$ & $96(90 \%)$ & $101(95)$ \\
\hline & $\mathrm{p}<0.0001$ & $\mathrm{p}<0.001$ & $\mathrm{p}<0.0001$ & $\mathrm{p}<0.0001$ \\
\hline
\end{tabular}

CT, chemotherapy 
Table 3. Changes in Median NLR and MRD Subtype Post Chemotherapy

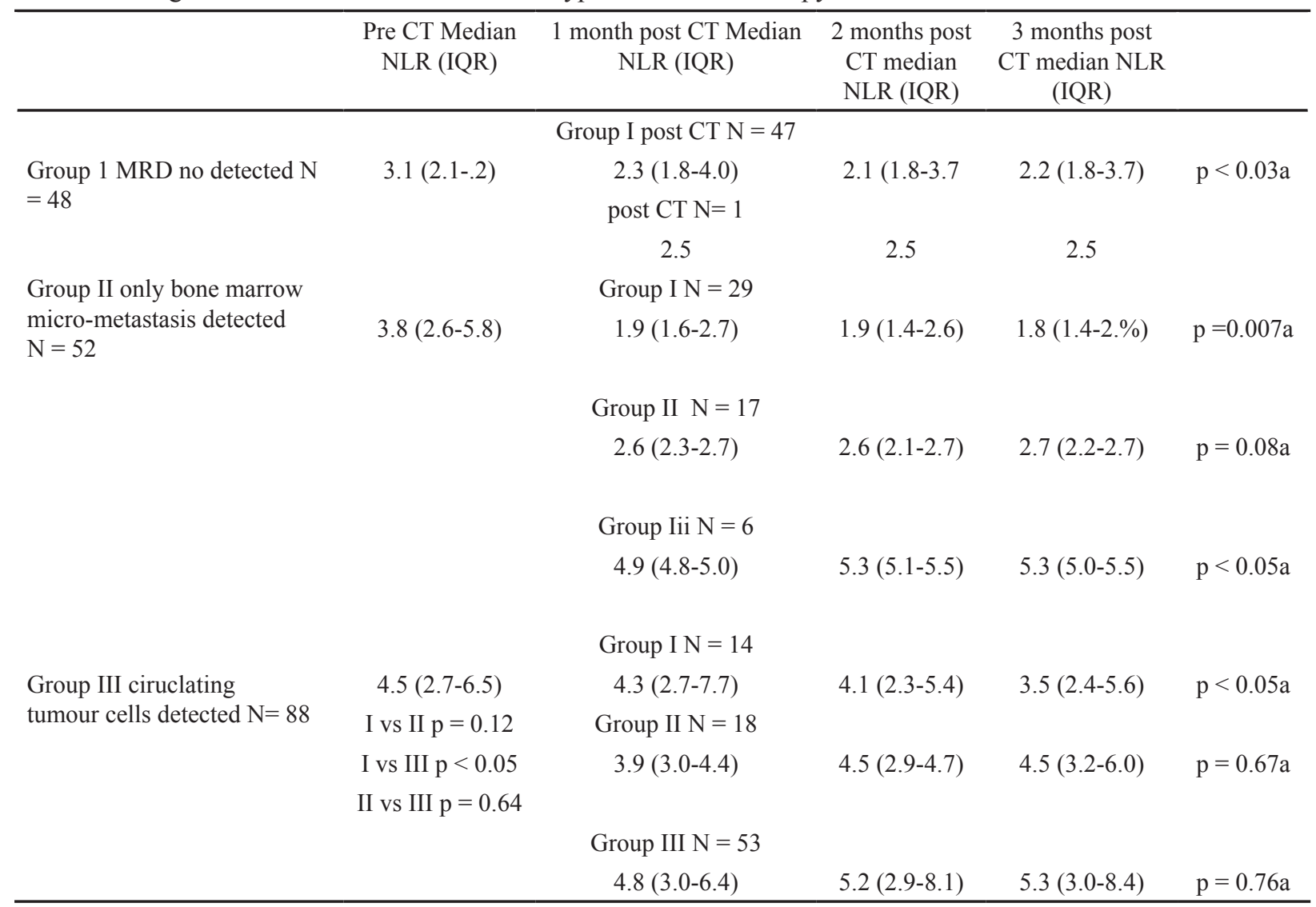

CT, chemotherapy; NLR, neutrophilto lymphocyte ratio; IQR, interquartile range; a, Mann-Whitney test pre-chemotherapy versus 3 minths post chemotherapy

patients in Group I pre-treatment had a significantly lower NLR post-chemotherapy $(\mathrm{p}<0.03)$. Those patients initially in Group II had a significant decrease in the NLR when they changed to Group I post treatment $(p=0.007)$ and

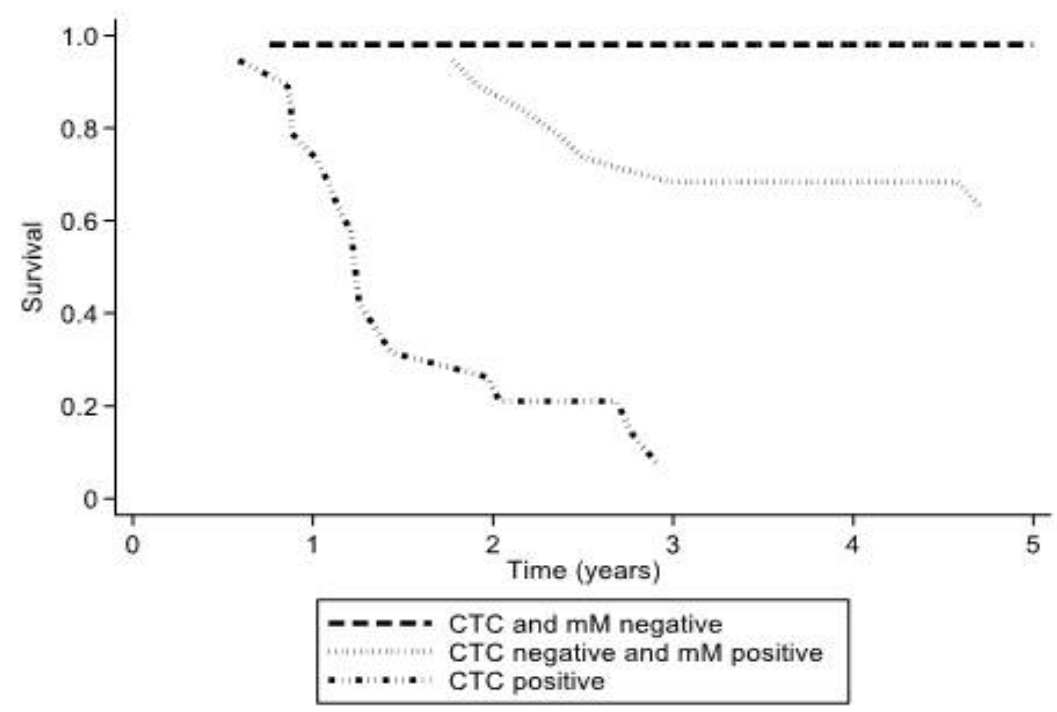

\begin{tabular}{|l|l|l|}
\hline MRD sub-type post CT & $\begin{array}{l}\% \text { Observed five year } \\
\text { survival }(95 \% \mathrm{Cl})\end{array}$ & RMST (95\% Cl) years \\
\hline $\begin{array}{l}\text { Group ICTC and mM }(-) \\
\mathrm{N}=90\end{array}$ & $88(66-96)$ & $4.6(4.2-5.0)$ \\
\hline $\begin{array}{l}\text { Group II CTC }(-) \mathrm{mM}(+) \\
\mathrm{N}=36\end{array}$ & $56(31-75)$ & $4.1(3.4-4.7)$ \\
\hline Group III CTC $(+) \mathrm{N}=59$ & $6(0.5-25)$ & $1.3(0.9-1.9)$ \\
\hline
\end{tabular}

Figure 5. Kaplan-Meier Survival Curves for up to Five Years of Follow-up According to Minimal Residual Disease Sub-Types after Chemotherapy for Stage III Colon Cancer. CTC, circulating tumour cell; mM, micro-metastasis; RMST, restricted mean survival time; CI, confidence interval; CT, chemotherapy. 
Table 4. Change in the Frequency of NLR with Differing Cut-Offvalues and Minimal

\begin{tabular}{|c|c|c|c|c|c|c|c|c|}
\hline \multicolumn{6}{|c|}{ Pre CT NLR } & \multicolumn{3}{|c|}{ Post CT NLR } \\
\hline & $<3.0$ & $<4.0$ & $<5.0$ & Group Post CT & & $<3.0$ & $<4.0$ & $<5.0$ \\
\hline \multirow{4}{*}{$\begin{array}{l}\text { Group I no } \\
\text { MRD detected } \\
N=48 \quad \mathrm{~N}(5)\end{array}$} & $22(46)$ & $30(63)$ & 34 )71) & Gp $1 \mathrm{~N}=47$ & i month & $33(70)$ & $36(77)$ & $38(79)$ \\
\hline & & & & & 2 months & $33(70)$ & 36 I77) & $40(85)$ \\
\hline & & & & & 3 months & $33(70)$ & $38(81)$ & $44(94)$ \\
\hline & & & & & & $\mathrm{p}=0.71$ & $\mathrm{p}=0.77$ & $\mathrm{p}=0.89$ \\
\hline \multirow{12}{*}{$\begin{array}{l}\text { Group II only } \\
\text { bone marrrow } \\
\text { micrometasta- } \\
\text { sis } \mathrm{N}=52\end{array}$} & $18(35)$ & $28(54)$ & $32(62)$ & Gp I N=29 & 1 month & $24(83)$ & $28(97)$ & $28(97)$ \\
\hline & & & & & 2 months & $24(83)$ & $28(97)$ & $28(97)$ \\
\hline & & & & & 3 months & $24(83)$ & $28(97)$ & $28(97)$ \\
\hline & & & & & & $\mathrm{p}=0.001$ & $\mathrm{p}<0.001$ & $\mathrm{p}<0.001$ \\
\hline & & & & Gp IIN = 17 & 1 month & $12(71)$ & $14(82)$ & $16(94)$ \\
\hline & & & & & 2 months & $15(88)$ & $15(88)$ & $16(94)$ \\
\hline & & & & & 3 months & $16(94)$ & $16(94)$ & $16(94)$ \\
\hline & & & & & & $\mathrm{p}=0.02$ & $\mathrm{p}<0.001$ & $\mathrm{p}<0.001$ \\
\hline & & & & Gp III N=6 & 1 month & $1(17)$ & $2(33)$ & $3(50)$ \\
\hline & & & & & 2 months & $1(17)$ & $2(33)$ & $2(33)$ \\
\hline & & & & & 3 months & $1(17)$ & $1(17)$ & $1(17)$ \\
\hline & & & & & & $\mathrm{p}=0.57$ & $\mathrm{p}=0.19$ & $\mathrm{p}=0.09$ \\
\hline \multirow{13}{*}{$\begin{array}{l}\text { Group III } \\
\text { circukating } \\
\text { tumour cells } \\
\text { detected } \mathrm{N}=88\end{array}$} & $28(32)$ & $40(46)$ & $48(55)$ & Gp $1 \mathrm{~N}=17$ & 1 month & $11(65)$ & $14(82)$ & $15(88)$ \\
\hline & & & & & 2 months & $11(65)$ & $14(82)$ & $15(88)$ \\
\hline & & & & & 3 months & $12(71)$ & $14(82)$ & $15(88)$ \\
\hline & & II $p=0,15$ & & & & $\mathrm{p}=0.006$ & $\mathrm{p}=0.01$ & $\mathrm{p}=0.02$ \\
\hline & & II $p=0.88$ & & & & & & \\
\hline & & $\mathrm{I} p=0.34$ & & Gp II N =18 & 1 month & $11(61)$ & $14(78)$ & $16(89)$ \\
\hline & & & & & 2 months & $11(61)$ & $14(78)$ & $16(89)$ \\
\hline & & & & & 3 months & $11(61)$ & $14(78)$ & $16(89)$ \\
\hline & & & & & & $\mathrm{p}=0.04$ & $\mathrm{p}=0.03$ & $\mathrm{p}=0.02$ \\
\hline & & & & Gp III N = 53 & 1 month & $12(23)$ & $26(49)$ & $28(53)$ \\
\hline & & & & & 2 months & $13(25)$ & $26(49)$ & $28(53)$ \\
\hline & & & & & 3 months & $13(25)$ & $26(49)$ & $28(53)$ \\
\hline & & & & & & $\mathrm{p}=0.32$ & $\mathrm{p}=0.82$ & $\mathrm{P}=0.99$ \\
\hline
\end{tabular}

significantly increased when they changed to Group III $(p<0.05)$. There was no significant change in the NLR in those patients who remained in Group II $(\mathrm{p}=0.08)$. The results are shown in Table 3. Comparing the median NLR post-chemotherapy for all Group I Patients versus all Group II and Group III patients, revealed the following results; There was no significant difference between Group I and Group II 2.4 (IQR 1.8-3.9) versus 2.7 (IQR 2.2-4.2) respectively ( $\mathrm{p}=0.59)$, whereas the median values for both Group I and II were significantly lower when compared with Group III (median NLR 4.8 (IQR 3.4-5.9) ( $\mathrm{p}=0.04$ and $\mathrm{p}=0.02$ respectively).

Change in frequency of NLR with differing cut-off values and Minimal Residual Disease subtype after chemotherapy

The frequency of a significantly lower NLR after chemotherapy was associated with an improvement in the MRD sub-type. Patients initially in Group II MRD and who changed to Group I MRD after chemotherapy had a significantly higher frequency of a low NLR $(p<0.001)$; interestingly patients who remained in Group
II MRD also had a significantly higher frequency of a low NLR $(p<0.001)$. In initially Group III patients, those patients improving MRD status had a significantly higher frequency of a low NLR $(p<0.04)$. The results are shown in Table 4. .

\section{Discussion}

There are conflicting reports on the value of the NLR in patients with stage III colon cancer, in part because there is no consensus cut-off value to define high or low risk of relapse and secondly at what point in follow up should the NLR be evaluated. Cha reported that a NLR of 3.0 was unable to predict outcome following FOLFOX chemotherapy for stage III colon cancer, however the NLR was taken prior to surgery (Cha et al., 2019). Li et al., (2014) reported that a NLR $>3.0$ pre-surgical resection was associated with a poorer overall survival (16) as did Maliletzis et al., (2014), while Pedrazzani et al., (2017) reported a cut-off value of NLR of $>3.5$ was associated with a poorer PFS and OS. However, we suggest that 
post-treatment NLR is a better prognostic marker for the following reasons; firstly the effect of the primary tumour on inflammatory markers has been removed. The primary tumour not only is the source of disseminated tumour cells but also is also responsible for preparing the pre-metastatic niche (Zhao et al., 2018) and modulating the immune system (Whiteside. 2013). Even in stage IV disease removal of the primary tumour has been reported to increase survival and decrease or normalized immune dysfunction (Gulack et al., 2016). Secondly, of those reports comparing pre with post resection NLR levels, it was concluded that changes in the NLR or the post-resection value was a better prognostic marker than pre-surgical values (Guo et al., 2018; Ashizawa et al., 2020). In stage II colon cancer treated with surgery alone, in patients with MRD negative colon cancer, the NLR significantly decreased after resection suggesting that removal of the primary tumour has an effect on immune function and thus represents a more accurate measure of immune function and possible future relapse (Murray et al., 2020). In thus study post-resection MRD negative patients had a significantly lower median NLR compared with CTC positive MRD patients, but had a similar median NLR value compared to micro-metastasis only MRD positive patients.

As for timing for the determination of the NLR, we choose three time periods after completing the six cycles of chemotherapy. This was based on the timing of routine controls post chemotherapy rather than a scientific reason. Hayama et al., (2019) reported that he NLR taken seven days post surgical resection was associated with prognosis. However, the level of the systemic inflammatory response after surgery is reported to return to baseline after 21-90 days (Chan et al., 2018), in this context the prechemotherapy NLR samples were taken between 28-42 days post surgery and as such minimized the effect of post-resection inflammation. In terms of the NLR post FOLFOX there are no longitudinal studies to suggest the optimal timing of blood sampling. FOLFOX is monthly, permitting the recovery haematological prior to the next cycle of chemotherapy but there are no long-term studies evaluating the haematological recovery. Similarly for the evaluation of MRD we choose the one-month after completing chemotherapy as the sampling time based on studies of neoadjuvant therapy in breast cancer patients (Mathiesen et al., 2012), although there is no standardized sampling time of bone marrow.

After chemotherapy, patients who did not relapse had a significantly better immune function as compared with those patients who relapsed, both compared with baseline values and between groups, both in terms of median NLR and frequency of a low NLR for the three cut-off values used. This could be because chemotherapy eliminates the disseminated tumour cells and this improves immune function, or per se has immune-modulating effects that also may eliminate tumour cells. The fact that the median NLR and frequency of a high NLR decreased in MRD negative patients after chemotherapy suggests that there was an immune-modulatory effect of FOLFOX, assuming that there was no MRD within the limits of the methodology. A change in the median NLR and frequency of a low NLR after chemotherapy was associated with changes in MRD prognostic group. In terms of MRD a higher median NLR or high-risk cut-off group was associated with a worse MRD prognostic group, the worst NLR results being present in Group III CTC positive patients. In mouse models using B16F1 cancer cells the authors concluded that CTCs could induce systemic inflammation and in consequence promoted the metastatic colonization of disseminated tumour cells. In addition they further reported that the function of neutrophils could be converted to a pro-tumour subtype in the presence of CTCs, in the absence of neutrophils (due to in vivo depletion) CTCs were unable to promote metastasis even though they induced systemic inflammation ( $\mathrm{Li}$ et al., 2017). Furthermore that changes in neutrophil subtype to a pro-tumour phenotype is associated with the triggering of growth in dormant micro-metastasis as a result of angiogenesis activation (Luo et al., 2016). Although our study was not designed to classify sub-types of neutrophils, our results suggest that the higher NLR seen in CTC positive patients (Group III) in comparison con the NLR seen in micro-metastasis positive patients (Group II) may explain in part the difference in progression free survival curves. Patients in Group III have a high risk of early relapse, suggesting a more aggressive disease and differed from Group II patients who were at risk for late relapse. The progression free survival curves for the first two years were similar between Group II patients and patients MRD negative (Group I). This may explain why one-month post chemotherapy there was no significant difference between Group I and II patients with respect to the median NLR, but both Groups had a significantly lower NLR than that found in Group III patients. It was beyond this study to determine if after two years the NLR in Group II patients started to increase or if there were CTCs present. This could be an early sign of pending disease progression.

One important result is that patients who changed to a better MRD subgroup had a significant decrease in the NLR and vice-versa. It was no possible to determine if chemotherapy improved the immune function and there improved MRD status or that improved MRD status resulted in improved immune function. As mentioned previously in mouse models there is a dynamic interaction between disseminated and circulating tumour cells and the immune system. In stage II colon cancer treated by surgery alone a higher NLR was associated with a worse MRD subtype and worse disease-free progression (Murray et al., 2020). The Kaplan-Meier curves for each MRD subtype were similar to those reported in this study, CTC positive patients having a high risk of early relapse and those with only micro-metastasis of late relapse.

The study has several limitations; firstly we used a non-commercial method for CTC detection. CTC detection is method dependent and the frequency and cur-off values to define a positive test vary. In localized colon cancer the EpCAM (Epithelial Cell Adhesion Molecule) based CellSearch ${ }^{\circledR}$ system has been reported to be positive in $5-25 \%$ of patients (Tsai et al., 2016), compared with $80 \%$ reported with the size based Metacell ${ }^{\circledR}$ system (Eliasova et al., 2017). Comparing CellSearch ${ }^{\circledR}$ 
with RT-PCR, CTCs were detected in $75 \%$ versus $20 \%$ of cases respectively and only in $14 \%$ of patients when using a gene mutation analysis (Gervasoni et al., 2011; Bahnassy et al., 2018). The detection method used in this study would not detect CEA negative CTCs and bone marrow micro-metastasis but is relatively cheap and while it is 10-100 folds less sensitive than RT-PCR it could use in the routine laboratory of a general hospital. It is possible that clinical utility is more important that sensitivity, a lowerlimit of CTC detection for predicting future relapse has not been established. Ultra sensitive detection methods may over-estimate clinically important MRD and detecting every cancer cell may not be important. Using RT-PCR in patients post allogeneic bone marrow transplantation for leukaemia, very small numbers of leukemic cells can be detected even though the patients remain in remission for many years; the leukemic cells surviving for many years before being eliminated by host immune systems (Cross. 1998). To decrease the potential differences in observer variability we used a positive/negative classification rather than define a specific cut-off value of CTCs/sample.

Secondly, the study includes a limited number of patients and thus further multicentre studies are required to confirm these findings. Finally the definition of NLR needs to be standardized and the optimal timing for blood and bone marrow sampling.

Conclusions: 1) MRD after chemotherapy determines patient outcome. 2) immune function as measured by the NLR is associated with MRD subtypes. 3) changes in immune function post chemotherapy are associated with changes in MRD subtype. 4) neither baseline MRD subtype or NLR predict the effect of chemotherapy on MRD. The results warrant further studies with a larger patient population.

\section{Author Contribution Statement}

Concept - N.P.M.; Design - N.P.M. RV, SA; Supervision -N.P.M., RV., SA.; Resources - N.P.M.; Materials - N.P.M., S.A., RV; Data Collection and/or Processing - RV., DH., S.A.; MR,; Analysis and/or Interpretation -N.P.M., S.A, RV.,; Literature Search-DH, SA; MR,; Writing Manuscript-NPM,; RV,; Critical review-SA, DH,; MR,; Final Approval- NPM,; RV,;SA,;DH,; MR.

\section{Acknowledgements}

\section{Funding Statement}

Funded by the Western Metropolitan Health Authority. The research grant paid for direct costs of the study, the funding source did not participate in the design, collection, analysis or interpretation of the data, in the writing of the report, its conclusions or decision to submit the paper for consideration to be published. The corresponding author participated actively in the study, had full access to all the study data and had final responsibility for the decision to submit the article for publication.

The authors wish to thank Mrs Ana Maria Palazuelos for her help in the redaction of this manuscript.

\section{Ethical Considerations}

The study was approved by the health authorites ethics and science committee and fully complied with the Declaration of Helsinki and Chilean law on patient's rights. All patients provided written informed consent prior to their participation.

Due to Chilean law on patients rights and confidentiality the data are not available for general study purposes.

\section{Conflicts of Interest}

The authors report no conflicts of interest.

\section{References}

Absenger G, Szkandera J, Stotz M, et al (2013). Preoperative neutrophil to lymphocyte ratio predicts clinical outcome in patients with stage II and III colon cancer. Anticancer Res, 33, 4591-4.

Ashizawa N, Furuya S, Katsutoshi S, et al (2020). Clinical significance of dynamic neutrophil lymphocyte ratio changes in patients with colorectal cancer. Anticancer Res, 40, 2311-7.

Bahnassy AA, Salem SE, Mohanad M, et al (2018). Prognostic significance of CTCs in Egyptian non-metastatic colorectal cancer detection: (flow-cytometry, CellSearch, quantitative real-time PCR and cytomorphology. Exp Mol Pathol, 106, 90-101.

Borgen E, Naume B, Nesland JM, et al (1999). Standardization of the immunocytochemical detection of cancer cells in bone marrow and blood, Establishment of objective criteria for the evaluation of immunostained cells. Cytotherapy, 5, 377-88.

Borazan E, Balik AA, Bozdag Z, et al (2017). Assessment of the relationship between neutrophil lymphocyte ratio and prognostic factors in non-metastatic colorectal cancer. Turk J Surg, 33, 185-9.

Cha YJ, Park EJ, Baik SH, Lee KY, Kang J (2019). Clinical significance of tumor-infiltrating lymphocytes and neutrophil-to-lymphocyte ratio in patients with stage III colon cancer who underwent surgery followed by FOLFOX chemotherapy. Sci Rep, 9, 11617.

Chan JCY, Diakos CL, Chan DLH, et al (2018). A longitudinal investigation of inflammatory markers in colorectal cancer patients perioperatively demonstrates benefit in serial measurement. Ann Surg, 267, 1119-25.

Cross NC (1998). Minimal residual disease in chronic myeloid leukaemia. Hematol Cell Ther, 40, 224-8.

Eliasova P, Pinkas M, Kolostova K, Gurlich R, Bobek V (2017). CTCs in different stages of colorectal cancer. Folia Histochemica et Cytobiologica, 55, 1-5.

Gervasoni A, Sandri MT, Nascimbeni R, et al (2011). Comparison of three distinct methods for the detection of CTCs in colorectal cancer patients. Oncol Rep, 25, 1669-703.

Gmeiner WH (2020). Fluoropyridime modulation of anti-tumor immune response-prospects for improved colorectal cancer treatment. Cancer, 12, 1641.

Gulack BC, Nussbaum DP, Keenan JE, et al (2016). Surgical resection of the primary tumor in stage IV colorectal cancer without metastasectomy is associated with improved overall survival compared with chemotherapy/radiation therapy alone. Dis Colon Rectum, 59, 299-305.

Guo D, Han A, Jing W, et al (2018). Preoperative to postoperative change in neutrophil to lymphocyte ratio predict survival in colorectal cancer patients. Future Oncol, 14, 1187-96.

Hayama T, Hashiguchi Y, Okada Y, et al (2019). Significance of the 7 th postoperative day neutrophil to lymphocyte ratio in colorectal cancer. Int $J$ Colorectal Dis, http//:doi. 
org:10.1007/s00384-019-03463-3.

Kaneko M, Nozawa H, Sasaki K, et al (2012). Elevated neutrophil to lymphocyte ratio predicts poor prognosis in advanced colorectal cancer patients receiving oxaliplatin based chemotherapy. Oncology, 82, 261-8.

Li X, Slayton WB (2013). Molecular mechanisms of platelet and stem cell rebound after 5-fluorouracil treatment. Exp Hematol, 41, 635-45.

Li MX, Liu XM, Zhang XF, et al (2014). Prognostic role of neutrophil to lymphocyte ratio in colorectal cancer: a systemic review and meta-analysis. Int $J$ Cancer, 134, 2403-12.

Li YC, Zou JM, Luo C, et al (2017). Circulating tumour cells promote the metastatic colonization of disseminated carcinoma cells by inducing systemic inflammation. Oncotarget, 8, 28418-30.

Luo J, Feng XX, Luo C, et al (2016). 14,15-EET induces the infiltration and tumor promoting function of neutrophils to trigger the growth of minimal dormant metastasis. Oncotarget, 7, 43324-36.

Maliietzis G, Giacometti M, Askari A, et al (2014). A preoperative neutrophil to lymphocyte ratio of 3 predicts disease-free survival after curative elective colorectal cancer surgery. Ann Surg, 260, 287-92.

Mathiesen RR, Borgen E, Renolen A, et al (2012). Persistence of disseminated tumor cells after neoadjuvant treatment for locally advanced brest cancer predicts poor survival. Breast Cancer Res, 14, r117.

Murray NP, Villalon R, Orrego S, Guzman E (2020). The association of the neutrophil to lymphocyte ratio with the presence of minimal residual disease and outcome in patients with Stage II colon cancer treated with surgery alone. Colorectal Dis, http//:doi.org:10.1111/codi.15438.

Pedrazzani C, Mantovani G, Fernandes E, et al (2017). Assessment of neutrophil to lymphocyte ratio, platelet to lymphocyte ratio and platelet count as predictors of long term outcome after R0 resection for colorectal cancer. Sci Rep, 7, 1494.

Salvatore L, Imperatori M, Arnoldi E, et al (2020). Management of patients with early-stage colon cancer: guidelines of the Italian Medical Oncology Association. ESMO open, 5, e001001.

Shibutani M, Maeda K, Nagahara H, et al (2013). A high preoperative neutrophil to lymphocyte count is associated with poor survival in patients with colorectal cancer. Anticancer Res, 33, 3291-4.

Shibutani M, Maeda K, Nagahara H, et al (2015). The prognostic significance of a postoperative systemic inflammatory response in patients with colorectal cancer. World J Surg Oncol, 13, 194.

Shingawa T, Tanaka T, Nozawa H, et al (2018). Comparison of the guidelines for colorectal cancer in Japan, the USA and Europe. Ann Gastroenterol Surg, 2, 6-12.

Song Y, Yang Y, Gao P, et al (2017). The preoperative neutrophil to lymphocyte ratio is a superior indicator of prognosis compared with other inflammatory biomarkers in resectable colorectal cancer. BMC Cancer, 17, 744.

Stojanovska V, Prakash M, McQuade R, et al (2019). Oxaliplatin treatment alters systemic immune response. Biomed Res Int, http//:doi.org:10.1155/2019/4650695.

Tsai WS, Chen JS, Shao HJ, et al (2016). CTC count correlates with colorectal neoplasm progression and is a prognostic marker for distant metastasis in on-metastatic patients. Sci Rep, http://doi.org: 10.1038/srep/24517.

Whiteside TL (2013). Immune modulation of T-cell and NK cell activities by tumour derived exosomes. Biochem Soc Trans, 41, 245-51.
Yasui K, Shida D, Nakamura Y, et al (2021). Postoperative but not preoperative, inflammation based prognostic markers are prognostic factors in stage III colorectal cancer patients. $\mathrm{Br}$ $J$ Cancer, 124, 933-41.

Zhao H, Achreja A, Iessi E, et al (2018). The key role of extracelular vesicles in the metastatic process. Biochem Biophys Acta, 869, 64-77.

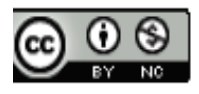

This work is licensed under a Creative Commons AttributionNon Commercial 4.0 International License. 